\title{
Electrical switching of an antiferromagnet
}

\author{
P. Wadley, ${ }^{1, \#, *}$ B. Howells, ${ }^{1, *}$ J. Železný ${ }^{2,3}$ C. Andrews, ${ }^{1}$ V. Hills, ${ }^{1}$ \\ R. P. Campion, ${ }^{1}$ V. Novák,${ }^{2}$ K. Olejník,${ }^{2}$ F. Maccherozzi, ${ }^{4}$ S. S. Dhesi, ${ }^{4}$ \\ S. Martin, ${ }^{5}$ T. Wagner, ${ }^{5}$ J. Wunderlich, ${ }^{2,5}$ \\ F. Freimuth, ${ }^{6}$ Y. Mokrousov, ${ }^{6}$ J. Kuneš, ${ }^{2}$ \\ J. S. Chauhan, ${ }^{1}$ M. J. Grzybowski, ${ }^{1,7}$ A. W. Rushforth, ${ }^{1}$ \\ K. W. Edmonds, ${ }^{1}$ B. L. Gallagher, ${ }^{1}$ T. Jungwirth ${ }^{2,1}$ \\ ${ }^{1}$ School of Physics and Astronomy, University of Nottingham, \\ Nottingham NG7 2RD, United Kingdom \\ ${ }^{2}$ Institute of Physics, Academy of Sciences of the Czech Republic, \\ Cukrovarnická 10, 16200 Praha 6, Czech Republic \\ ${ }^{3}$ Faculty of Mathematics and Physics, Charles University, \\ Ke Karlovu 3, 12116 Prague 2, Czech Republic \\ ${ }^{3}$ Diamond Light Source, Chilton, Didcot, Oxfordshire, OX11 0DE, United Kingdom \\ ${ }^{5}$ Hitachi Cambridge Laboratory, J. J. Thomson Avenue, \\ Cambridge CB3 0HE, United Kingdom \\ ${ }^{6}$ Peter Grünberg Institut and Institute for Advanced Simulation, \\ Forschungszentrum Jülich and JARA, 52425 Jülich, Germany \\ ${ }^{7}$ Institute of Physics, Polish Academy of Sciences, \\ al. Lotnikow 32/46, 00-681 Warsaw, Poland \\ * Authors contributed equally to this work. \\ \#To whom correspondence should be addressed; E-mail: Peter.Wadley@ nottingham.ac.uk.
}

Louis Néel pointed out in his Nobel lecture that while abundant and interesting from a theoretical viewpoint, antiferromagnets did not seem to have any applications. Indeed, the alternating directions of magnetic moments on indi- 
vidual atoms and the resulting zero net magnetization make antiferromagnets hard to control by tools common in ferromagnets. Remarkably, Néel in his lecture provides the key which, as we show here, allows us to control antiferromagnets by electrical means analogous to those which paved the way to the development of ferromagnetic spintronics applications. The key noted by Néel is the equivalence of antiferromagnets and ferromagnets for effects that are an even function of the magnetic moment. Based on even-in-moment relativistic transport phenomena, we demonstrate room-temperature electrical switching between stable configurations combined with electrical read-out in antiferromagnetic CuMnAs thin film devices. Our magnetic memory is insensitive to and produces no magnetic field perturbations which illustrates the unique merits of antiferromagnets for spintronics.

Information technology devices are either charge-based or spin-based. Among the weaknesses of charge-based devices is that perturbations such as ionizing radiation can lead to uncontrolled charge redistributions and, as a consequence, to data loss. All commercial spin-based devices rely on one principle in which the opposite magnetic moment orientations in a ferromagnet (FM) represent the zeros and ones (1). This technology is behind memory applications ranging from kilobyte magnetic stripe cards to megabyte magnetoresistive random access memories (MRAMs) and terabyte computer hard disks. Since based on spin, the devices are robust against charge perturbations. Moreover, they are non-volatile and, compared to the charge flash memory, MRAM offers short read/write times suitable for the main random-access computer memories. However, the FM moments can be unintentionally reoriented and the data erased by disturbing magnetic fields generated externally or internally within the memory circuitry. The vision behind our work are future non-volatile information technologies based on antiferromagnets (AFMs) that are robust against charge and magnetic field perturbations, and which utilize 
other advantages of AFMs compared to FMs including the invisibility of data stored in AFMs to external magnetic probes, ultrafast spin dynamics, and the broad range of metal, semiconductor, or insulator materials with room-temperature AFM order (2-7).

Several roots from micro-device, relativistic quantum physics, and materials research had to meet to provide us with the means to experimentally demonstrate the concept of a roomtemperature AFM memory with electrical writing and reading. In FMs the energy barrier separating stable directions of ordered spins is due to the magnetic anisotropy energy. It is an even function of the magnetic moment which, following Néel's general principle noted in his Nobel lecture (8), implies that the magnetic anisotropy and the corresponding memory functionality are readily present also in AFMs (9).

The magneto-transport counterpart of the magnetic anisotropy energy is the anisotropic magnetoresistance (AMR). In the early 1990's, the first generation of FM MRAM micro-devices used AMR for the electrical read-out of the memory state (10). AMR is an even function of the magnetic moment which, following again Néel's principle, implies its presence in AFMs (11). While AMR in AFMs was experimentally confirmed in several recent studies (12-17), efficient means for manipulating AFM moments have remained elusive.

External magnetic field, whose coupling is linear in the magnetic moment, favors parallel alignment of the moments, i.e., acts against the staggered exchange field in the AFM. On the other hand, current-induced even magnetic torques of the form $d \mathbf{M} / d t \sim \mathbf{M} \times(\mathbf{M} \times \mathbf{p})$, which are used for electrical writing in the most advanced FM spin-transfer-torque MRAMs (1), have been proposed to allow for a large angle reorientation of the AFM moments (18). In these antidamping-like torques $\mathbf{M}$ is the magnetic moment vector and $\mathrm{p}$ is the electrically injected carrier spin-polarization. Translated to AFMs, the effective field proportional to $\left(\mathbf{M}_{A, B} \times \mathbf{p}\right)$ which drives the antidamping-like torque $d \mathbf{M}_{A, B} / d t \sim \mathbf{M}_{A, B} \times\left(\mathbf{M}_{A, B} \times \mathbf{p}\right)$ on individual spin sublattices A and B is staggered, i.e., alternates in sign between the opposite spin sublattices. 
The staggered property of the effective current-induced field is the key for its strong coupling to the Néel order.

In FM spin-transfer-torque MRAMs, spin polarized carriers are injected into the free FM layer from a fixed FM polarizer by an out-of-plane electrical current driven through the FMFM stack. Reversible $180^{\circ}$ switching is achieved by reversing the polarity of the out-of-plane current. In analogy with FM spin-transfer-torque MRAMs, the above theory proposed for AFMs (18) assumes injection of the spin polarized carriers into the AFM from a fixed FM polarizer by out-of-plane electrical current driven in a FM-AFM stack. Unlike the FM-FM stack, the effect of the spin current injected from a FM to an AFM is independent of the current polarity. The AFM spin-axis can in principle be switched from the parallel to the perpendicular direction with respect to the magnetization of the FM polarizer but cannot be switched back electrically (18). Another limitation of the stack geometry with out-of-plane spin injection is that the absorption of carrier spins is limited to magnetic film thicknesses of the order of the spin diffusion length which in AFMs is typically on the nanometer scale (19).

Several of us have predicted (20) that relativistic quantum mechanics may offer staggered current induced fields which do not require external polarizers and act in bare AFM crystals. Instead of changing the current polarity, a reversible $90^{\circ}$ switching is facilitated by applying electrical currents along two orthogonal directions. The effect occurs in AFMs with specific crystal and magnetic structures for which the spin sublattices form space-inversion partners. Among these materials is a high Néel temperature AFM, tetragonal-phase CuMnAs, which we have recently synthesized in the form of single-crystal epilayers structurally compatible with common semiconductors, such as $\mathrm{Si}$ or $\mathrm{GaP}$ (21). Relativistic current-induced fields strongly coupled to the Néel order, combined with AFM CuMnAs epilayers and micro-device AMR, are the key elements that led to our demonstration of the electrical switching in an AFM memory resistor which we now describe in more detail. 
We first outline the basic theoretical considerations behind our experiments. Relativistic current-induced fields observed previously in broken inversion-symmetry FM crystals (22-30) can originate from the inverse spin galvanic effect (31-35) which is illustrated in Fig.1 A. For illustrative purposes we consider here that the spin-orbit term in the Hamiltonian of the system, which originates from broken inversion symmetry in the crystal, has a two-dimensional Rashba form with carrier spins aligned in the direction perpendicular to the momentum. The applied electrical current induces an asymmetric non-equilibrium distribution function of carrier states and, as a result, a non-zero carrier spin-polarization $\mathbf{p}$ aligns perpendicular to the applied current. In FMs, the non-equilibrium carrier spin density acts on magnetic moments as an effective magnetic field when carrier spins are exchange-coupled to the magnetic moments. The resulting field-like torque has a form $d \mathbf{M} / d t \sim \mathbf{M} \times \mathbf{p}$. When the sense of the inversion symmetry of the lattice reverses, the sign of the carrier spin polarization and of the corresponding effective field also reverses for the same orientation of the applied current, as illustrated in Fig. 1B.

The full lattice of our CuMnAs crystal, shown in Fig. 1C, has an inversion symmetry; the center of inversion of the lattice is at an interstitial position, highlighted by the green ball in the figure. This implies that the mechanism described in Figs. 1A,B will not generate a net current-induced spin-density when integrated over the entire crystal. However, $\mathrm{Mn}$ atoms form two sublattices depicted in Fig. 1C in red and purple whose local environment has broken inversion symmetry and the two Mn sublattices form inversion partners. The inverse spin galvanic mechanisms of Figs. 1A,B will generate locally non-equilibrium spin polarizations of opposite signs on the inversion-partner Mn sublattices. For these staggered fields to couple strongly to the Néel order it is essential that the inversion-partner Mn sublattices coincide with the two spin-sublattices A and B of the AFM ground-state (20). The resulting spin-sublattice torques have the form $d \mathbf{M}_{A, B} / d t \sim \mathbf{M}_{A, B} \times \mathbf{p}_{A, B}$ where, in analogy with the even torques, the effective field proportional to $\mathbf{p}_{A}=-\mathbf{p}_{B}$ acting on the spin-sublattice magnetizations alternates in 

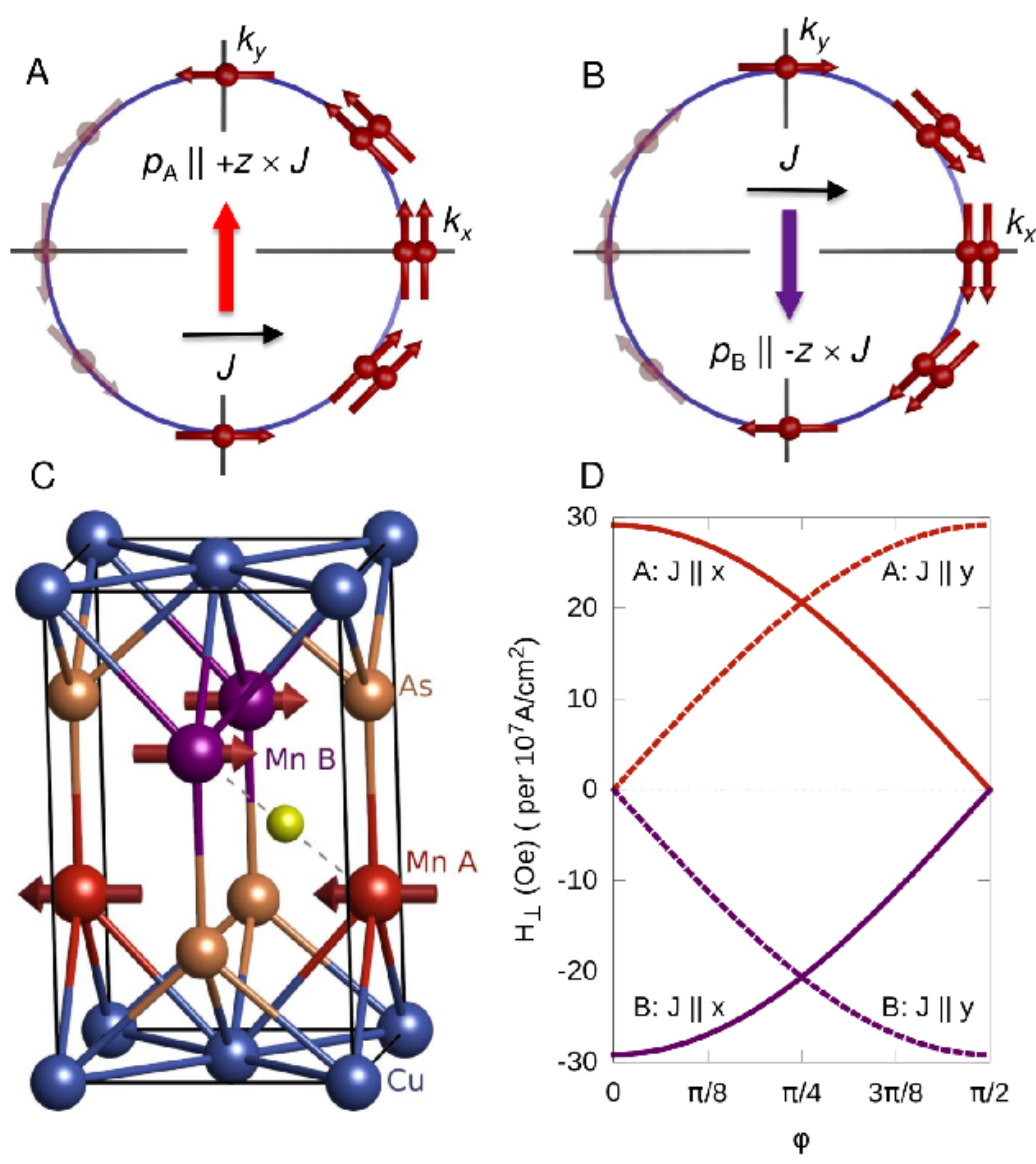

Figure 1: (A) Schematic of the inverse spin galvanic effect in a model inversion asymmetric Rashba spin texture (red arrows). $k_{x, y}$ are the in-plane momentum components. The nonequilibrium redistribution of carriers from the left side to the right side of the Fermi surface results in a net in-plane spin polarization (thick red arrow) along $+z \times J$ direction, where $J$ is the applied current (black arrow). (B) Same as (A) for opposite sense of the inversion asymmetry resulting in a net in-plane spin polarization (thick purple arrow) along $-z \times J$ direction. (C) CuMnAs crystal structure and AFM ordering. The two Mn spin-sublattices A and B (red and purple) are inversion partners. This and panels (A) and (B) imply opposite sign of the respective local current induced spin polarizations, $p_{A}=-p_{B}$, at spin-sublattices A and B. The full CuMnAs crystal is centrosymmetric around the interstitial position highlighted by the green ball. (D) Microscopic calculations of the components of the current induced field transverse to the magnetic moments at spin-sublattices $\mathrm{A}$ and $\mathrm{B}$ as a function of the magnetic moment angle $\varphi$ measured from the $\mathrm{x}$-axis ([100] crystal direction). The electrical current is applied along the $\mathrm{x}$ and $\mathrm{y}$-axes. 
sign between the two sublattices. This again implies the strong coupling to the Néel magnetic order. As shown in Fig. 1C, CuMnAs crystal and magnetic structures fulfil these symmetry requirements (21).

To quantitatively estimate the strength of the staggered current induced field we performed microscopic calculations based on the Kubo linear response formalism. The electronic structure of CuMnAs was obtained by the Jülich density functional theory code FLEUR which is an implementation of the full-potential linearized augmented plane-wave method (36). In Fig. 1D we plot the resulting components of the in-plane current induced field transverse to the magnetic moments at spin-sublattices A and B as a function of the in-plane magnetic moment angle $\varphi$ measured from the $\mathrm{x}$-axis ([100] crystal direction). The electrical current of $10^{7} \mathrm{Acm}^{-2}$ is applied along the $\mathrm{x}$-axis or the $\mathrm{y}$-axis.

The calculations confirm the desired opposite sign of the current induced field on the two spin-sublattices. They also highlight the expected dependence on the magnetic moment angle which implies that the AFM moments will tend to align perpendicular to the applied current. For reversible electrical switching between two stable states, the setting current pulses can therefore be applied along two orthogonal in-plane cubic axes of CuMnAs. We also emphasize that the amplitude of the effect seen in Fig. 1D is comparable to typical current induced fields applied in FMs, suggesting that CuMnAs is a favorable material for observing the effect in an AFM.

Our experiments were conducted on epitaxial films of the tetragonal phase of CuMnAs, which is a member of a broad family of high-temperature I-Mn-V AFM compounds $(6,7,21)$. We have observed the electrical switching and readout effects that we describe below in more than 20 devices fabricated from 5 different CuMnAs films, with thickness ranging from $40 \mathrm{~nm}$ to $80 \mathrm{~nm}$, grown on either $\mathrm{GaP}$ or GaAs substrates. The electrical data presented here were obtained on a $46 \mathrm{~nm}$ epilayer on lattice-matched $\mathrm{GaP}(001)$, whose transmission electron microscopy image shown in Fig. 2A demonstrates excellent crystal quality and chemical or- 
der (21). Consistent with the AFM order of the CuMnAs film, superconducting quantum interference device (SQUID) measurements in Fig. 2B show only the diamagnetic background of the sample substrate. Neutron diffraction confirmed collinear AFM order with a Néel temperature $T_{N}=480 \mathrm{~K}(21,37)$. The CuMnAs film is metallic with a room-temperature sheet resistivity of $160 \mu \Omega \mathrm{cm}$.

In Figs. 2C,D we demonstrate the electrical writing in an electronic CuMnAs microchip device. The optical microscopy image of the device and the electrical writing and reading geometry are shown in Fig. 2C. The sample is placed in a cryostat at a stable temperature of $273 \mathrm{~K}$ inferred from the temperature calibration of the resistivity of the CuMnAs film. In the measurements shown in Fig. 2D, three successive $50 \mathrm{~ms}$ writing pulses of amplitude $J_{\text {write }}=$ $4 \times 10^{6} \mathrm{Acm}^{-2}$ are applied alternately along the [100] crystal direction of CuMnAs (black arrow in Fig. 2C and corresponding black points in Fig. 2D) and along the [010] axis (red arrow in Fig. $2 \mathrm{C}$ and corresponding red points in Fig. 2D). Note that $J_{\text {write }}=4 \times 10^{6} \mathrm{Acm}^{-2}$ is the current density in the central region of the device obtained from finite element modelling for the applied current of $90 \mathrm{~mA}$ driven through the $28 \mu \mathrm{m}$ wide writing arms of the device. The reading current $J_{\text {read }}$ is applied in this experiment along the [110] in-plane diagonal and resistance signals, $R_{\perp}$, transverse to $J_{\text {read }}$ are recorded $10 \mathrm{~s}$ after each $J_{\text {write }}$ pulse. (A constant offset is subtracted from $R_{\perp}$.)

According to our theory prediction, the [100]-directed writing pulses are expected to set a preference for domains with AFM spin-axis along the [010] direction (black double-arrow in Fig. 2C) and the [010]-directed pulses for domains with AFM spin-axis along the [100] direction (red double-arrow in Fig. 2C). Consistent with the domain reconfiguration picture, successive $J_{\text {write }}$ pulses in one direction increase the amplitude of the read-out $R_{\perp}$ signal of one sign and pulsing in the orthogonal direction increases the amplitude of $R_{\perp}$ of the opposite sign. As seen in Fig. 2D, all the AFM memory states can be written reproducibly. The signals are independent 

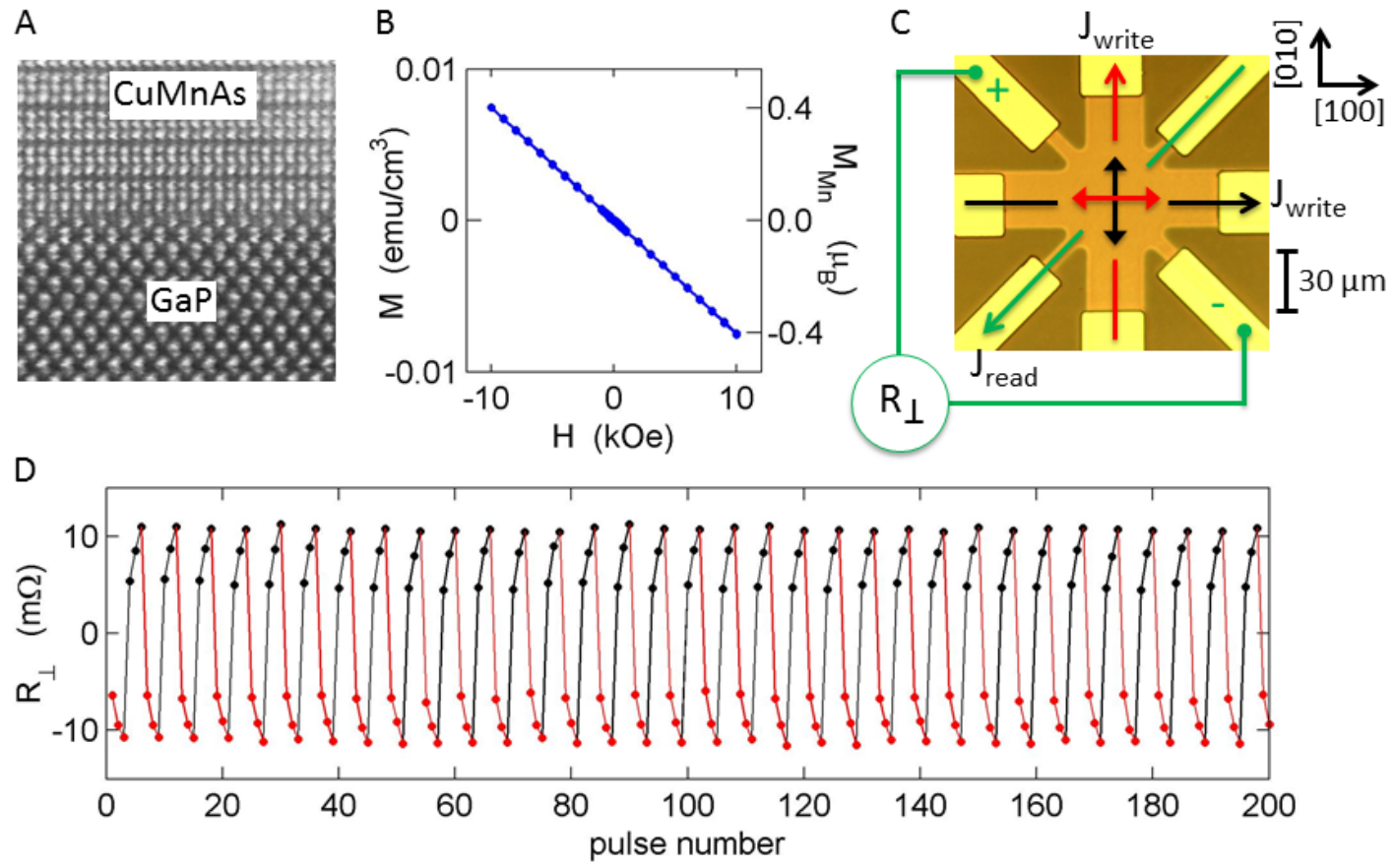

Figure 2: (A) Scanning transmission electron microscopy image of CuMnAs/GaP in the [100][001] plane. (B) Magnetization versus applied field of an unpatterned piece of the $\mathrm{CuMnAs} / \mathrm{GaP}$ wafer measured by SQUID magnetometer. (C) Optical microscopy image of the device and schematic of the measurement geometry. (D) Change in the transverse resistance after applying three successive $50 \mathrm{~ms}$ writing pulses of amplitude $J_{\text {write }}=4 \times 10^{6} \mathrm{Acm}^{-2}$ alternatively along the [100] crystal direction of CuMnAs (black arrow in panel $\mathrm{C}$ and corresponding black points in panel D) and along the [010] axis (red arrow in panel $\mathrm{C}$ and corresponding red points in D). The reading current $J_{\text {read }}$ is applied along the [110] axis and transverse resistance signals $R_{\perp}$ are recorded $10 \mathrm{~s}$ after each writing pulse. A constant offset is subtracted from $R_{\perp}$. Measurements were done at sample temperature of $273 \mathrm{~K}$. 
of the polarity of the writing current which is expected for the current-induced switching in AFMs. We note that the amplitude of the switching current applied in our AFM memory is significantly lower than in the first observation of spin-orbit torque switching in ferromagnetic metals, where $100 \mathrm{MA} \mathrm{cm}^{-2}$ pulses were used to reverse magnetization in a Pt/Co bilayer (30).

In Figs. 3A,B we explore in more detail the domain reconfiguration by applying a series of fifty $J_{\text {write }}$ pulses of varying length and amplitude along the [010] direction (red points) and [100] direction (black points) at $273 \mathrm{~K}$. The data, which again show highly reproducible switching patterns, illustrate that the imbalance in the domain populations increases with the length and amplitude of the writing pulses and tends to saturate with the increasing number of pulses. Since in these measurements heating of the central region of the device can reach tens of degrees during the writing pulses we did not explore the switching behavior further beyond the pulse lengths and amplitudes shown in Figs. 3A,B. More intense biasing in our device design can lead to irreproducible pulsing characteristics or device failure due to structural changes.

Apart from the absolute $R_{\perp}$ values, we also indicate in Figs. 3A,B relative values $R_{\perp} / \bar{R}$ of the signal, where $\bar{R}$ is the longitudinal resistance $R$ averaged over the different states set by the writing pulses along the [100]/[010] directions. Below we will associate $R_{\perp} / \bar{R}$, reaching $0.2 \%$, with the AFM AMR. Before turning the attention to the origin of the electrical read-out signal we present in Fig. 4 x-ray magnetic linear dichroism (XMLD) measurements recorded at beamline I06 of Diamond Light Source, which confirm the picture of the current induced domain reconfiguration by the applied writing pulses.

In the left panel of Fig. 4A we show the optical microscope image of the cross-structure used in the XMLD measurements. The $10 \mu \mathrm{m}$ wide arms of the cross-structure are patterned along the $[100] /[010]$ directions. A photoemission electron microscopy (PEEM) image of the central region of the structure is presented in the middle panel of Fig. 4A. The room temperature AFM domain structure before applying writing pulses is illustrated in the right panel of Fig. 4A 

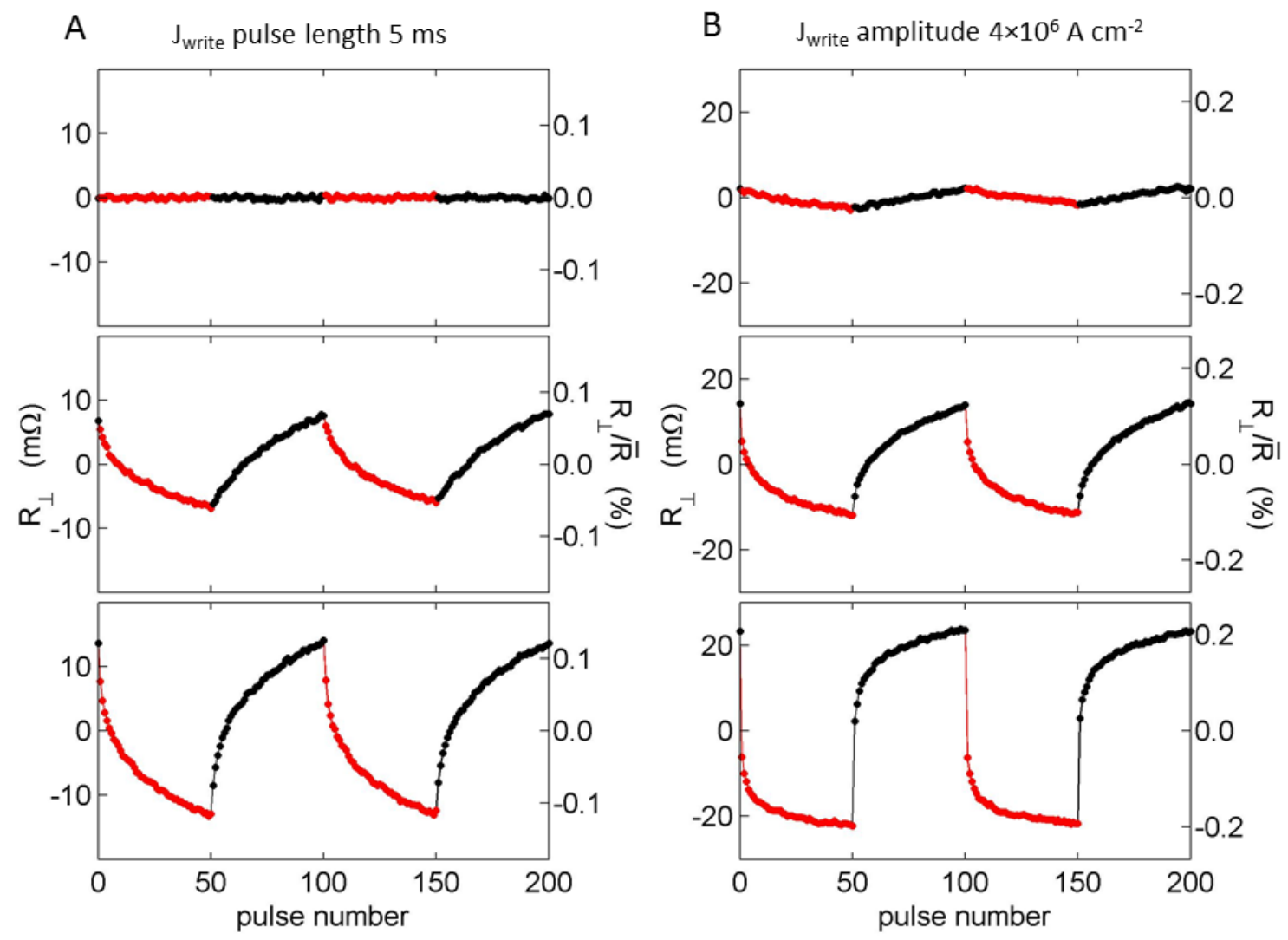

Figure 3: Transverse resistance after successive writing pulses along the [100] axis (black points) and [010] axis (red points) for different current amplitudes (A) or pulse lengths (B). $R_{\perp}$ is recorder $10 \mathrm{~s}$ after each writing pulse. $\bar{R}$ is the average of the longitudinal resistance $R$. Measurements were done at sample temperature of $273 \mathrm{~K}$. A constant offset is subtracted from $R_{\perp}$. 
where we show an XMLD-PEEM image of a part of the central region. Images were taken with X-rays at the $\mathrm{Mn} L_{3}$ absorption edge incident at $16^{\circ}$ from the surface along the [100] axis. Xrays were $s$-polarized, i.e., along the [010] axis. By taking the difference of the absorption at the Mn $L_{3}$ edge valley (blue dashed line in Fig. 4C) minus the absorption at the $\mathrm{Mn} L_{3}$ edge peak (red dashed line in Fig. 4C), the images are sensitive to the AFM domain structure due to the XMLD at the Mn $L_{3}$ edge. The XMLD-PEEM image taken in the virgin state before applying current pulses shows sub-micron scale texture which largely disappears for $p$-polarized x-rays, indicating that it is due to AFM domains with in-plane spin orientation. The populations of domains with [100] and [010] oriented AFM spins are comparable. (The dark rings in Fig. 4A middle and the corresponding bright rings in Fig. 4B are due to residual photoresist from the sample processing).

In Fig. 4B we show room temperature XMLD-PEEM images after applying three $50 \mathrm{~ms}$ pulses of amplitude $6 \times 10^{6} \mathrm{Acm}^{-2}$. In order to directly compare in one PEEM measurement the effect on the domain structure of the current pulses along the [100] and [010] directions we applied the writing current between the right and top arms, as shown in the left panel of Fig. 4A. After the current pulses, there is a reconfiguration and coalescence of domains. At the top arm of the cross, where the writing current direction is along the [010] axis, bright domains have enlarged at the expense of dark domains, as further highlighted in the detailed image in the middle panel of Fig. 4B. Vice versa, dark domains have enlarged at the expense of bright domains at the right arm where the writing current direction is along the [100] axis. This is highlighted in the detailed image in the right panel of Fig. 4B.

According to theory expectations for the current induced spin-orbit fields, dark domains have AFM spins along the [010] axis and bright domains along the [100] axis. To confirm this we have calibrated the sign of the XMLD signal in CuMnAs by comparing a measured XMLD spectrum with $a b$ initio calculations (38). In the top panel of Fig. 4C we plot the Mn 


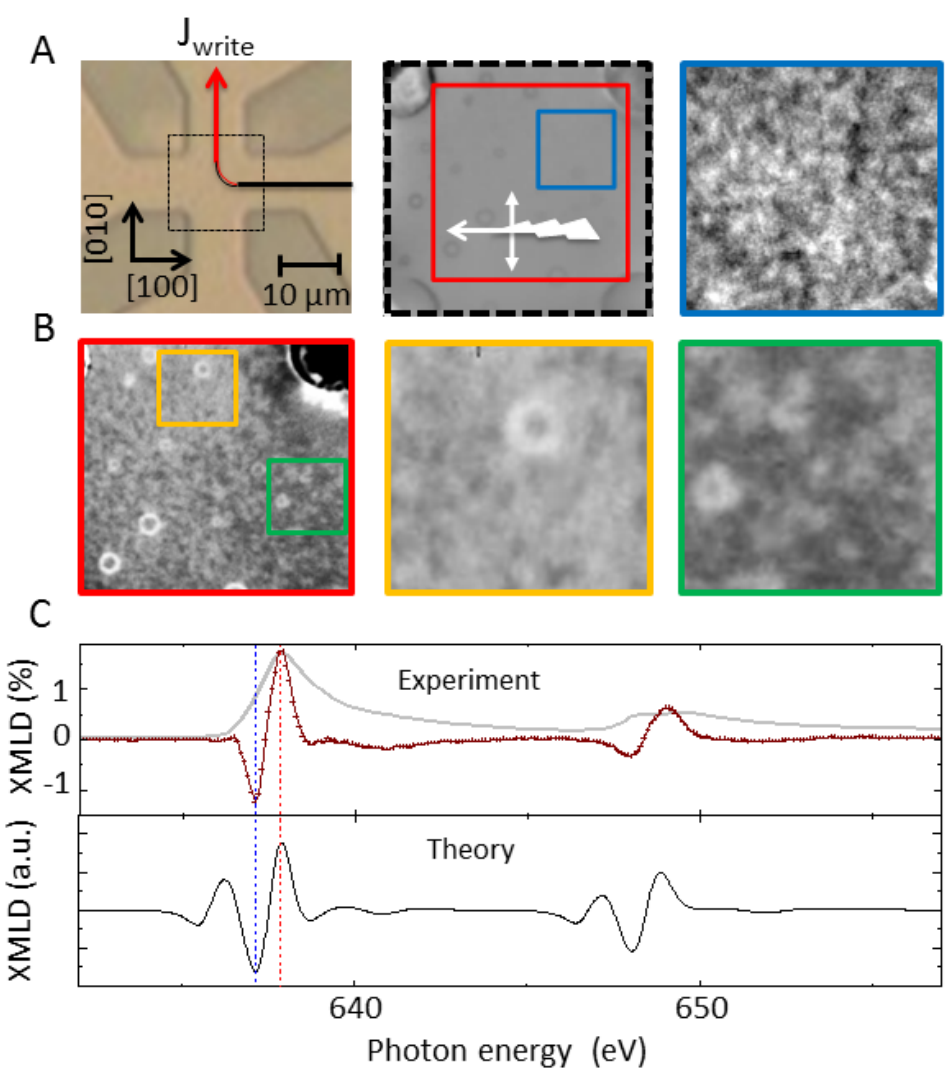

Figure 4: (A) Left: Optical microscope image of the cross-structure used in the XMLD-PEEM experiment. The black/red arrow labelled $J_{\text {write }}$ shows the path of the current pulses. Middle: $15 \mu \mathrm{m} x$-ray absorption image of the central region (dashed box in the left A panel). Images were taken with x-rays at the $\mathrm{Mn} L_{3}$ absorption edge incident at $16^{\circ}$ from the surface along the [100] axis (white lightning arrow). X-rays were $s$-polarized, i.e., along the [010] axis (white double-arrow). Right: $5 \mu \mathrm{m}$ XMLD-PEEM image of a part of the central region (blue box in the middle A panel) before pulsing. (B) Left: $13 \mu \mathrm{m}$ XMLD-PEEM image of the central region (red box in the middle A panel) after three $50 \mathrm{~ms}$ pulses of amplitude $6 \times 10^{6} \mathrm{Acm}^{-2}$. Middle: Same as left panel with the detail within a $4 \mu \mathrm{m}$ square at the top arm (yellow box in the left B panel). Right: Same as left panel with the detail within a $4 \mu \mathrm{m}$ square at the right arm (green box in the left B panel). (C) Top panel: A. Mn $L_{2,3}$ XMLD spectrum for a uniaxial 10nm CuMnAs film, obtained as the absorption for normal incidence $\mathrm{x}$-rays with polarization parallel to the [010] crystalline axis minus the absorption for the polarization perpendicular to the [010] crystalline axis. Red and blue dashed lines indicate the positions of the Mn $L_{3}$ absorption edge peak and valley used for generating the XMLD-PEEM images in A and B. The absorption spectrum is shown by the grey line. Bottom panel: Calculated XMLD spectrum for this geometry, defined as the absorption for AFM moments parallel to the x-ray polarization, minus the absorption for AFM moments perpendicular to the x-ray polarization. Experiments were performed at room temperature. 
$L_{2,3}$ XMLD spectrum measured in spatially averaged total electron yield mode for a $10 \mathrm{~nm}$ thick CuMnAs film at room temperature. Films of this thickness have a uniaxial easy magnetic axis due to the interface anisotropy induced by the GaP substrate, resulting in an approximately single domain state. The spectrum was obtained as the absorption for normal incidence x-rays with polarization parallel to the [010] crystalline axis, minus the absorption for the polarization perpendicular to the [010] crystalline axis. In the bottom panel of Fig. 4C we plot the calculated XMLD spectrum, defined as the absorption for AFM moments parallel to the $\mathrm{x}$-ray polarization, minus the absorption for AFM moments perpendicular to the x-ray polarization. The positions of the principal peaks at both the $L_{3}$ and $L_{2}$ edges are in good agreement with the experimental data. From the comparison of measured and calculated XMLD spectra in Fig. 4C, we infer that bright domains in the PEEM images in Figs. 4B correspond to AFM moments aligned perpendicular to the x-ray polarization axis, i.e., along the [100] axis while dark domains correspond to AFM moments aligned parallel to the $\mathrm{x}$-ray polarization axis, i.e., along the [010] axis. This is consistent with the expected orthogonal alignment of the current induced spin-orbit field, and of the corresponding AFM spin axis, to the applied current direction.

We now proceed to establishing the AMR origin of the electrical reading signal by analyzing the symmetry of the measured resistances for different probe current directions. The temperature dependence of the measured AMR and its comparison to ab initio AMR calculations will provide us with another confirmation of the orthogonal alignment of the AFM moments to the writing current direction. In Fig. 5 we show switching data obtained at the sample temperature of $150 \mathrm{~K}$. In these lower-temperature experiments, we applied five successive $275 \mathrm{~ms}$ pulses of amplitude $J_{\text {write }}=4.5 \times 10^{6} \mathrm{Acm}^{-2}$ along the [100] or [010] axis to obtain comparable signals as in the higher-temperature measurements. Side by side we compare the transverse resistance signal with the longitudinal signal, $\Delta R / \bar{R}$, where $\Delta R=R-\bar{R}$. Each row in Fig. 5 corresponds to a different axis along which we apply the probe current $J_{\text {read }}$. From top to bottom, 
the reading current is applied along the crystal axis [110], [110], [100], and [010].

Consistent with the AMR symmetry, the transverse signals (also known as the planar Hall effect) are detected for the AFM spin-axes angle set towards $\pm 45^{\circ}$ from the probe current and the transverse signal flips sign when the probe current is rotated by $90^{\circ}$. The corresponding longitudinal signals vanish in this geometry. For AFM spin-axes set towards $\pm 90^{\circ}$ from the probe current the transverse and longitudinal AMR signals switch places compared to the $\pm 45^{\circ}$ angle between the probe-current and spin-axis. This is again consistent with the AMR symmetries, and the AMR nature of the electrical signals is further confirmed by comparable amplitudes of the transverse and longitudinal signals. We note that apart from the stable AMR signals, the longitudinal resistances show an additional time-dependence which is due to the cooling of the sample after the writing pulses. These isotropic changes in $R$ correspond to a temperature change of a fraction of a Kelvin over the probing time-interval.

We confirmed these AMR symmetries also in higher-temperature measurements. However, the AMR changes sign between the higher and lower-temperature data as seen when comparing the transverse resistance signals in Fig. 2 and 3 with the corresponding measurements in the first row of Fig. 5. The change in sign of the AMR is further confirmed in Fig. 6A. Here the states were first set at $150 \mathrm{~K}$ under the same writing conditions as in Fig. 5. Then the AMR was measured while increasing the sample temperature, without resetting the states by the write pulses during the temperature sweep. The AMR sign in the device changes at $250 \mathrm{~K}$. To reconfirm this we performed additional measurements in which we set the states by the writing pulses and probed at temperatures shifted by $\pm 20 \mathrm{~K}$ from the transition point. We again detected the opposite sign of the AMR.

The temperature dependence of the AMR sign has been reported already in the early studies in FMs (39). We have performed transport calculations using the ab initio band structure and linear response theory to explore the temperature-dependent AMR in the CuMnAs AFM. The 
A
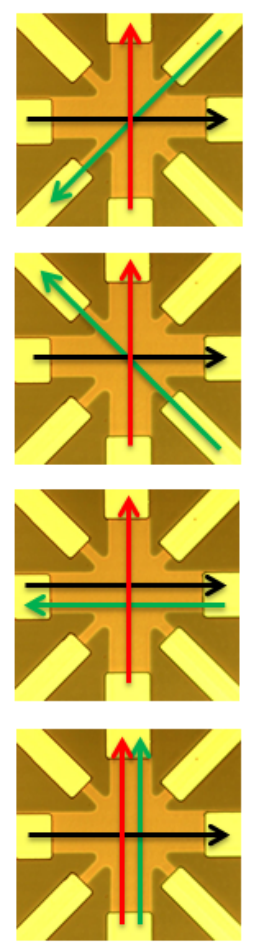

B
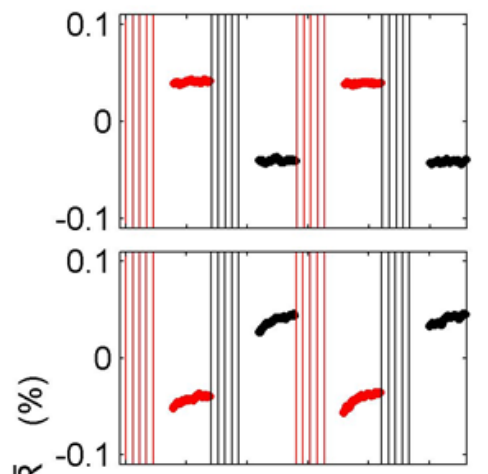

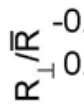
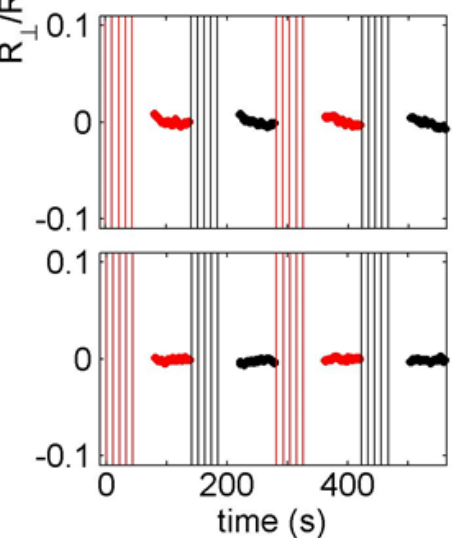

C
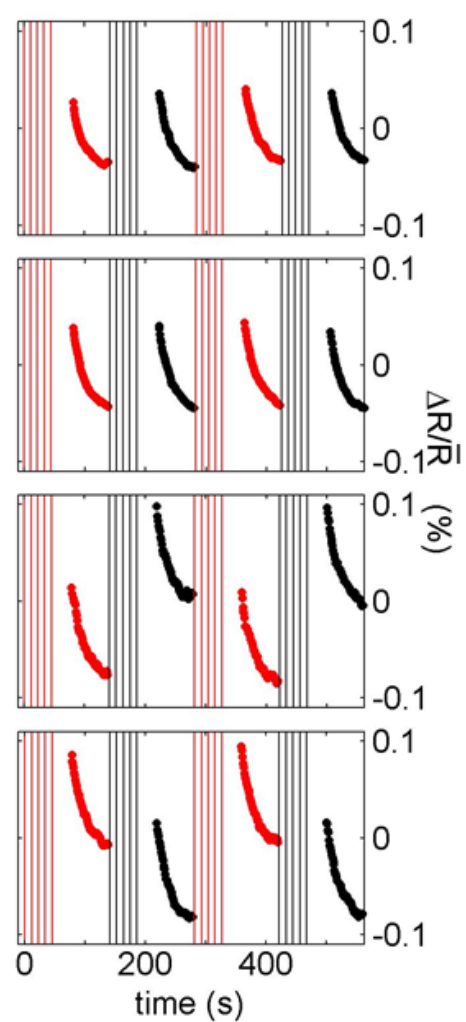

Fig. 5. (A) Optical microscopy image of the device and of the measurement geometries with different probe current directions (green arrows). The writing current directions are shown by black and red arrows. (B) Normalised transverse resistance $R_{\perp} / \bar{R}$ after five writing current pulses along the [100] axis (black) and five pulses along the [010] axis (red) for the reading current directions shown in (A). Vertical lines indicate the times of the pulses. The pulse length is $275 \mathrm{~ms}$ and amplitude $J_{\text {write }}=4.5 \times 10^{6} \mathrm{Acm}^{-2}$. Measurements were done at sample temperature of $150 \mathrm{~K}$. A constant offset is subtracted from $R_{\perp}$. (C) As for (B) but for the normalised longitudinal resistance change, $\Delta R / \bar{R}$ where $\Delta R=R-\bar{R}$. 
results are plotted, side by side, with the experimental temperature-dependent AMR in Fig. 6A. In the calculations, the temperature change is modelled by varying the quasiparticle spectral broadening $\Gamma$ between 100 and $300 \mathrm{meV}$. This corresponds to a range of theoretical sheet resistances of 150-200 $\mu \Omega \mathrm{cm}$ which approximately matches the experimental sheet resistance variation within the temperature interval of the AMR measurement in Fig. 6A. The spectral broadening is the only adjustable parameter in these ab initio calculations. While modelling the temperature effects on AMR by $\Gamma$ represents only a simplified, albeit commonly used description, it semiquantitatively captures the observed sign change and the overall magnitude of the AMR.

Directly from measurements and without further interpretation, the sign of the experimental AMR is expressed in terms of the perpendicular versus parallel configuration of the writing and reading currents, as highlighted in the left panel of Fig. 6A. On the other hand, the theoretical AMR is calculated by considering the perpendicular versus parallel configuration of the AFM spin axis and the reading current, as indicated in the right panel of Fig. 6A. By comparing the experimental and theoretical panels in Fig. 6A we can therefore infer the preferred AFM spin axis direction for the given writing current direction. The experimental and theoretical AMR signs match on the respective lower and higher temperature sides if the AFM spin axis aligns perpendicular to the writing current. This is consistent with the XMLD-PEEM results and with the theoretically predicted direction of the spin-orbit current induced fields.

In Fig. 6B we put our picture of the current induced switching in the CuMnAs microdevices under one more consistency test. AFMs are rather insensitive to external magnetic fields, however, there is an energetic preference for the AFM spin axis to align perpendicular to the magnetic field (8). Fig. 6B shows measurements in which we set at zero magnetic field each of the two states by ten $50 \mathrm{~ms}$ pulses with the writing current amplitude $J_{\text {write }}=4.5 \times 10^{6} \mathrm{Acm}^{-2}$. The experiments were performed in a magnetic cryostat at $305 \mathrm{~K}$. After setting the states at zero 
A

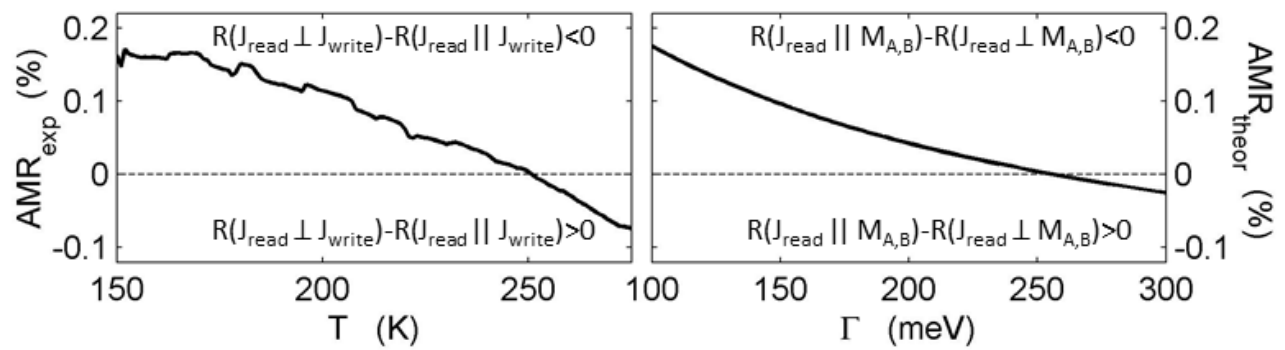

B
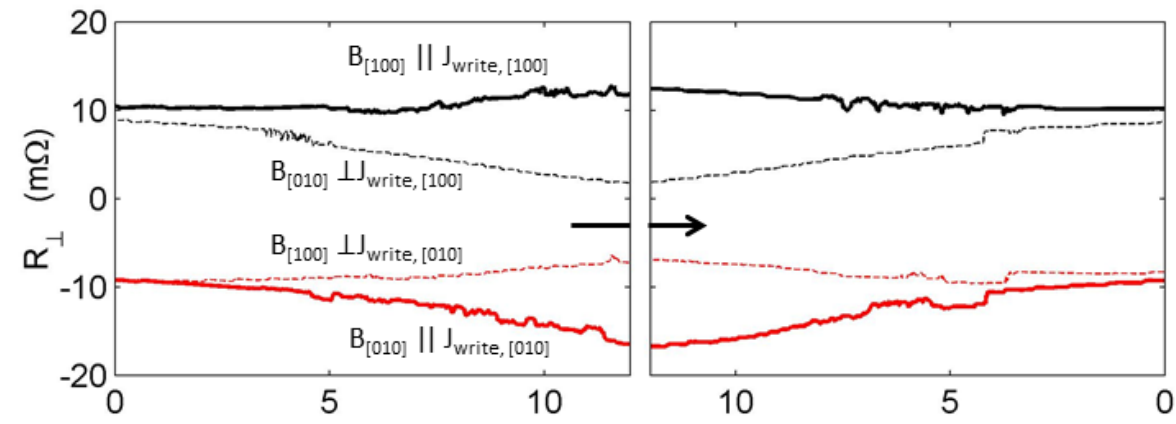

B ( $\mathrm{T})$

$\mathrm{C}$
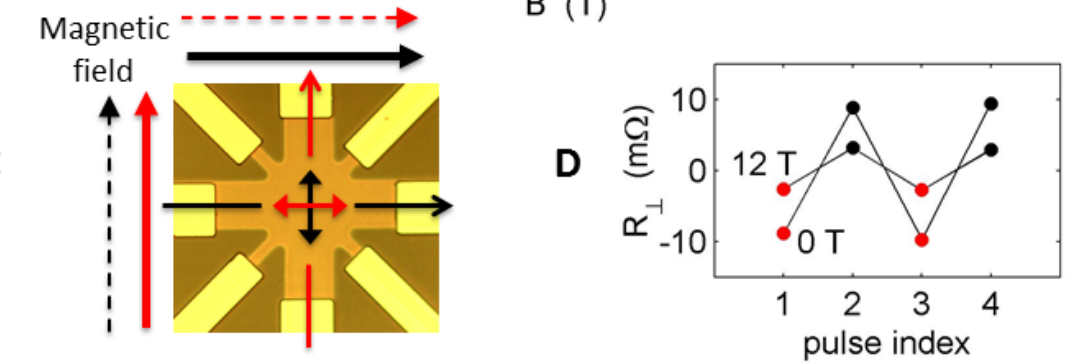

Figure 5: (A) Left: Experimental AMR obtained from the temperature-dependent $R_{\perp} / \bar{R}$ measured after applying the writing current pulses along the [100] or [010] axis at $150 \mathrm{~K}$. The sign of the experimental AMR is highlighted in the panel. Right: Theoretical AMR as a function of the quasiparticle scattering rate. The sign of the theoretical AMR is highlighted in the panel. (B) Dependence of $R_{\perp}$ on magnetic field swept from 0 to $12 \mathrm{~T}$ and back to 0 . The experiments were performed at $305 \mathrm{~K}$ after setting the states at zero field by ten $50 \mathrm{~ms}$ pulses with the writing current amplitude $J_{\text {write }}=4.5 \times 10^{6} \mathrm{Acm}^{-2}$. (C) Four experimental configurations used in the measurements in panel B. (D) Comparison of the two states set at zero magnetic field and at $12 \mathrm{~T}$. A constant offset is subtracted from $R_{\perp}$. 
field we swept the magnetic field first from 0 to $12 \mathrm{~T}$ and then back to 0 . We repeated the measurement in the four configurations shown in Fig. 6C; two in which the field and the writing current are parallel and the other two configurations with the orthogonal arrangement of the magnetic field and the writing current.

Consistent with the AFM nature of the states, even the $12 \mathrm{~T}$ field is not sufficient to overwrite the signal set by the writing current pulses at zero field. Nevertheless, the field induces sizable transient changes which enhance the signal when the magnetic field is parallel to the writing current and suppress the signal in the orthogonal configuration. This is again consistent with the current induced spin-orbit field, and consequently the AFM spin axis, aligning perpendicular to the writing current. In Fig. 6D we compare the read-out transverse resistances in states set by current pulses at either zero magnetic field or at $12 \mathrm{~T}$. The experiment further highlights that our AFM memory can be read and written by the staggered current induced fields in the presence of strong magnetic field perturbations.

To conclude, reorienting AFMs with an efficiency comparable to FMs requires a field compatible with the Néel magnetic order. Generating external magnetic fields with a sign alternating on the scale of a lattice constant at which moments alternate in AFMs has been considered unfeasible, hindering the applications of these abundant magnetic materials. We have succeeded in generating electrically such an alternating field and utilized its strong coupling to the Néel order for switching the CuMnAs AFM between configurations which are stable at ambient conditions and robust against magnetic field perturbations. The staggered field is generated electrically with comparable efficiency and by relativistic physics analogous to that which has driven recent major advancements in the FM spintronics research.

The staggered current induced fields we observe are not unique to CuMnAs. A high Néel temperature $\mathrm{AFM} \mathrm{Mn}_{2} \mathrm{Au}(40)$ is another example in which the spin-sublattices form inversion partners and where theory predicts large field-like torques of the form $d \mathbf{M}_{A, B} / d t \sim \mathbf{M}_{A, B} \times$ 
$\mathbf{p}_{A, B}$ with $\mathbf{p}_{A}=-\mathbf{p}_{B}(20)$. From microscopic density-functional calculations, we obtain a current-induced field of around 20 Oe per $10^{7} \mathrm{Am}^{-2}$ in $\mathrm{Mn}_{2} \mathrm{Au}$, which combined with its higher conductivity may make this a favorable system for observing current-driven AFM switching. AFMs which do not possess these specific symmetries can be alternatively interfaced with spinorbit coupled non-magnetic (NM) layers. A spin current will be injected into the AFM from the NM layer by an applied in-plane electrical current via the spin Hall effect, generating the antidamping-like torque $d \mathbf{M}_{A, B} / d t \sim \mathbf{M}_{A, B} \times\left(\mathbf{M}_{A, B} \times \mathbf{p}\right)(20)$. The same type of torque can be also generated by the spin-orbit Berry-curvature mechanism acting at the inversion-asymmetric AFM/NM interface or in bare AFM crystals with globally non-centrosymmetric unit cells like $\mathrm{CuMnSb}$ (20). Our experiments in CuMnAs combined with the prospect of other realizations of these relativistic non-equilibrium phenomena in AFMs brings us to the conclusion that AFMs are now ready to join the rapidly developing fields of basic and applied spintronics and to enrich this area of solid state physics and microelectronics by the range of unique characteristics of AFMs.

\section{References and Notes}

1. C. Chappert, A. Fert, F. N. Van Dau, Nat. Mater. 6, 813 (2007).

2. A. V. Kimel, A. Kirilyuk, A. Tsvetkov, R. V. Pisarev, T. Rasing, Nature 429, 850 (2004).

3. M. Fiebig, et al., J. Phys. D. Appl. Phys. 41, 164005 (2008).

4. T. Yamaoka, J. Phys. Soc. Japan 36, 445 (1974).

5. W. Zhang, et al., Phys. Rev. Lett. 113, 196602 (2014).

6. T. Jungwirth, et al., Phys. Rev. B - Condens. Matter Mater. Phys. 83, 1 (2011).

7. F. Máca, et al., J. Magn. Magn. Mater. 324, 1606 (2012). 
8. L. Néel, http://www.nobelprize.org/nobel_prizes/physics/laureates/1970/neel-lecture.pdf .

9. R. Y. Umetsu, a. Sakuma, K. Fukamichi, Appl. Phys. Lett. 89, 1 (2006).

10. J. Daughton, Thin Solid Films 216, 162 (1992).

11. A. B. Shick, S. Khmelevskyi, O. N. Mryasov, J. Wunderlich, T. Jungwirth, Phys. Rev. B 81, 212409 (2010).

12. B. G. Park, et al., Nat. Mater. 10, 347 (2011).

13. Y. Y. Wang, et al., Phys. Rev. Lett. 109, 137201 (2012).

14. X. Marti, et al., Nat. Mater. 13, 367 (2014).

15. I. Fina, et al., Nat. Commun. 5, 4671 (2014).

16. T. Moriyama, et al., http://arxiv.org/abs/1507.06138.

17. D. Kriegner et al., http://arxiv.org/abs/1508.04877.

18. H. V. Gomonay, V. M. Loktev, Phys. Rev. B 81, 144427 (2010).

19. R. Acharyya, H. Y. T. Nguyen, W. P. Pratt, J. Bass, J. Appl. Phys. 109, 5 (2011).

20. J. Železný, et al., Phys. Rev. Lett. 113, 157201 (2014).

21. P. Wadley, et al., Nat. Commun. 4, 2322 (2013).

22. B. Bernevig, O. Vafek, Phys. Rev. B 72, 033203 (2005).

23. A. Chernyshov, et al., Nat. Phys. 5, 656 (2009).

24. M. Endo, F. Matsukura, H. Ohno, Appl. Phys. Lett. 97, 222501 (2010). 
25. D. Fang, et al., Nat. Nanotechnol. 6, 413 (2011).

26. A. Manchon, S. Zhang, Phys. Rev. B 78, 212405 (2008).

27. I. M. Miron, et al., Nat. Mater. 9, 230 (2010).

28. U. H. Pi, et al., Appl. Phys. Lett. 97, 162507 (2010).

29. T. Suzuki, et al., Appl. Phys. Lett. 98, 142505 (2011).

30. I. M. Miron, et al., Nature 476, 189 (2011).

31. A. Y. Silov, et al., Appl. Phys. Lett. 85, 5929 (2004).

32. Y. K. Kato, R. Myers, A. Gossard, D. D. Awschalom, Phys. Rev. Lett. 93, 176601 (2004).

33. S. D. Ganichev, et al., arXiv:cond-mat/0403641 (2004).

34. J. Wunderlich, B. Kaestner, J. Sinova, T. Jungwirth, arXiv:cond-mat/0410295v1 (2004).

35. J. Wunderlich, B. Kaestner, J. Sinova, T. Jungwirth, Phys. Rev. Lett. 94, 047204 (2005).

36. F. Freimuth, S. Blügel, Y. Mokrousov, Phys. Rev. B 90, 174423 (2014).

37. V. Hills, et al., J. Appl. Phys. 117, 1 (2015).

38. J. Kuneš and P. M. Oppeneer, Phys. Rev. B 67, 024431 (2003).

39. T. McGuire and R. Potter, IEEE Trans. Magn. 11, 1018 (1975).

40. V. M. T. S. Barthem, C. V. Colin, H. Mayaffre, M.-H. Julien, D. Givord, Nat. Commun. 4, 2892 (2013). 
We acknowledge support from EU ERC Advanced Grant No. 268066, from the Ministry of Education of the Czech Republic Grant No. LM2011026, from the Grant Agency of the Czech Republic Grant no. 14-37427, from the UK EPSRC Grant No. EP/K027808/1, from the EU 7th Framework Programme under the project REGPOT-CT-2013-316014 (EAgLE), from HGF Programme VH-NG 513 and DFG SPP 1568, supercomputing resources at Jülich Supercomputing Centre and RWTH Aachen University, and Diamond Light Source for the allocation of beamtime under proposal number SI-12504W. We thank Christopher Nelson for providing the STEM measurement. 